\title{
Compensation of Load Dynamics for Admittance Controlled Interactive Industrial Robots using a Quaternion-based Kalman Filter
}

\author{
Saverio Farsoni ${ }^{1}$, Chiara Talignani Landi ${ }^{2}$, Federica Ferraguti ${ }^{2}$, Cristian Secchi ${ }^{2}$ and Marcello Bonfè ${ }^{1}$
}

\begin{abstract}
The paper describes a control architecture for industrial robotic applications allowing human/robot interactions, using an Admittance Control scheme and direct sensing of the human inputs. The aim of the proposed scheme is to support the operator of an industrial robot, equipped with a force/torque (F/T) sensor on the end-effector, during human/robot collaboration tasks involving heavy payloads carried by the robot. In these practical applications, the dynamics of the load may significatively affect the measurements of the $F / T$ sensor. Model-based compensation of such dynamic effects requires to compute linear acceleration and angular acceleration/velocity of the load, that in this work are estimated by means of a quaternion-based Kalman filter and assuming that the only available measurements come from the forward kinematics of the robot. Experimental results demonstrate the feasibility of the approach and its industrial applicability.
\end{abstract}

Index Terms-Industrial Robots; Physical Human-Robot Interaction; Compliance and Impedance Control.

\section{INTRODUCTION}

C OLLABORATION between humans and robots is increasingly desired in several application domains, including the production industry. As a result, a new generation of industry-oriented robots has been launched on the market (e.g. KUKA LBR iiwa). Such robots support physical Human-Robot Interaction (pHRI) by embedding advanced control functionalities, not available on standard industrial robots, including compliant control modes (from simple gravity compensation to Cartesian impedance control [1]) and contact forces/torques estimation, possibly exploiting direct joint torque sensing.

In pHRI applications, contact forces/torques must be available for human/robot collision detection and human intents awareness. The first feature can be achieved using modelbased observers and the so-called residuals analysis [2], [3]. However, observer-based solutions require accurate knowledge of the dynamic parameters of the robot (i.e. masses, inertia tensors, friction), which may not be precisely known for

Manuscript received: September 8, 2016; Revised December 3, 2016; Accepted December 27, 2016.

This paper was recommended for publication by Editor Wan Kyun Chung upon evaluation of the Associate Editor and Reviewers' comments. This work is supported by the project CTN01_00163_216730, funded by the Italian Ministry of Education, University and Research

${ }^{1} \mathrm{~S}$. Farsoni and M. Bonfè are with the Engineering Department, University of Ferrara, Italy (e-mail name.surname@unife.it).

${ }^{2}$ C. Talignani Landi, F. Ferraguti and C. Secchi are with the Department of Science and Methods for Engineering, University of Modena and Reggio Emilia, Italy (e-mail: name.surname@unimore.it).

Digital Object Identifier (DOI): xxxxxxxxxxxxx. standard industrial robots (i.e. not natively designed for human/robot collaboration), especially those with high gear ratios and non-negligible friction effects. To cope with these issues, a solution based on the frequency-domain analysis of motor currents is described in [4], but exploiting the KUKA Robot Sensor Interface (RSI), a feature not available on many other commercial systems. Detection of human intents and their isolation from accidental collisions is instead reported, among others, in [3] and [5]. The latter proposes a control effortbased approach that should not require a dynamic model of the robot, but only the possibility for a human to manually change its end-effector position. This approach is reasonable for the light and back-drivable haptic device used in the experiments of [5], but questionable for large industrial robots with high gear ratios.

In this work, we propose a control framework that allows to extend pHRI to a larger part of industrial applications, in which non-backdrivable manipulators with stiff position controllers are installed. In these contexts, a compliant control mode can be implemented installing a 6 degrees-of-freedom (DOF) force/torque $(\mathrm{F} / \mathrm{T})$ sensor on the end-effector of the robot and exploiting an admittance control scheme. In particular, we consider those applications in which the robot is carrying a significative payload (i.e. a manipulated object or a heavy tool) and humans interact by applying forces/torques on that load. This condition requires to take into account the load dynamics, to compensate its effects from $\mathrm{F} / \mathrm{T}$ sensor measurements and properly detect human inputs. Compensation of load dynamics has been previously addressed in [6], proposing an impedance control scheme. Impedance control, unlike the admittance control adopted here, requires knowledge of robot dynamics and the possibility to command joint torques, two features that are not available on most industrial manipulators. Direct force control with non-contact effects elimination, based on load velocities/accelerations estimated from joint encoder data and differential kinematics, is reported in [7], but this approach still require knowledge of robot intrinsic data (i.e. calibrated kinematic parameter). Others authors describe similar estimation schemes using sensor fusion and accelerometers/gyroscopes [8], with the drawbacks of additional costs for hardware and additional sensor calibration requirements.

The main contribution of this paper is a solution for the estimation of accelerations and velocities of the load, to be used for the compensation of non-contact effects from F/T measurements, that does not require any additional sensor in the robotic setup and assumes that neither dynamic nor 
kinematic parameters of the mechanical structure of the robot are known. It is only required that the low-level robot controller, whose user may not have knowledge of kinematic parameters, provides an accurate measure of the 6-DOF pose of its end-effector at a reasonable update frequency. The proposed solution relies on a novel quaternion-based Kalman filter, based on a second order differential model, that directly processes measurements of the end-effector pose.

Quaternion-based kinematic models have been largely exploited, especially in the aerospace domain [9], to implement attitude estimation Kalman filters [10]. Such filters fuse data from accelerometers/magnetometers, as an indirect measure of attitude (i.e. assuming fixed gravity vector and Earth magnetic field), data from gyroscopes as a direct measure of angular velocities and compute first order quaternion derivatives, to be integrated for attitude estimation. In our design, instead, we use the Kalman filtering approach for differentiation, up to the second order, of a known position and orientation of the robot end-effector. Similar approaches have been applied in human body tracking [11], [12], but still without considering second order derivatives. Even in [13], that describes quaternion-based Kalman filters embedding also the estimation of environmental F/T acting on a robotic tool, angular accelerations are not included in the estimated state, with the result that F/T estimation errors are larger than those presented in this paper. In next sections, we will define more precisely the statement of the problem that we aim to address and the proposed overall control scheme.

\section{INTERACTION CONTROL SCHEME}

Robotic applications requiring to regulate the interaction of the robot with its environment, but also to cope with both free motions and in contact phases, are suitable for the implementation of either impedance or admittance control schemes [1]. The latter is more adequate when the robot has a stiff and non-backdrivable mechanical structure or is made purposefully stiff by an inner motion control loop, which is the common case in industrial robotics. In this situation, it is possible to force the robot to behave compliantly in response to a human input, according to a given mass-damper interaction model, provided that the human/robot contact force is measurable and that the interaction model is integrated to compute the motion of a compliant frame, which is set as the reference pose to the inner control loop.

In the following, we will denote: a 6 -DOF pose as $\mathbf{P}$, expressed by a homogenous transformation matrix embedding a translation vector $\mathbf{p}$ and a rotation matrix $\mathbf{R}$; a $6 \mathrm{D}$ spatial velocity vector as $\mathcal{V}=[\mathbf{v}, \boldsymbol{\omega}]^{T}$, embedding linear and angular velocities; a $6 \mathrm{D}$ force/torque $(\mathrm{F} / \mathrm{T})$ vector as $\mathbf{F}=[\mathbf{f}, \boldsymbol{\tau}]^{T}$. With this notation, the interaction model for admittance control is expressed by the following differential equation:

$$
\mathbf{\Lambda}_{d} \dot{\mathcal{V}}_{r e f}+\mathbf{D}_{d} \mathcal{V}_{r e f}=\mathbf{F}_{c}
$$

in which $\boldsymbol{\Lambda}_{d}$ and $\mathbf{D}_{d}$ are $6 \times 6$ symmetric and positive definite inertia and damping matrices, properly tuned to obtain a stable and smooth behavior even in case of human-driven interactions [14]. Since the contact force/torque $\mathbf{F}_{c}$ is an input, Eq. 1 is used to compute (from its discrete-time equivalent) the incremental motion to be added to the reference pose $\mathbf{P}_{\text {ref }}$ commanded to the low-level robot controller. The contact input is assumed to be measured from a 6-DOF F/T sensor mounted on the robot wrist flange. Moreover, we want to address case studies in which the robot is used to help humans in the manipulation of heavy objects, rigidly attached to the robot after the F/T sensor as shown in Fig. 1.

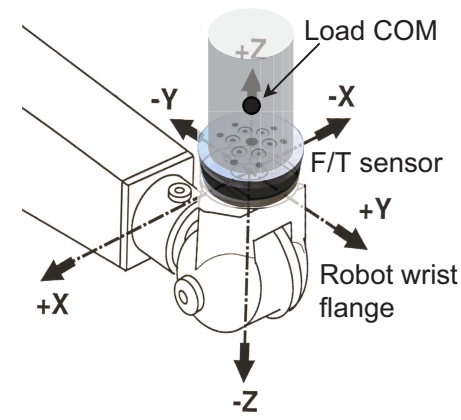

Fig. 1. Reference frames of F/T sensor and load

In this case, the load exerts a non-contact effect $\mathbf{F}_{n c}$ on the sensor, with both static (i.e. gravity) and dynamic terms (i.e. inertial, centrifugal/Coriolis). While the former could be computed and eliminated from F/T sensor measurements with a knowledge of the load mass, center-of-mass (COM) and orientation, the load inertia tensor and an estimate of linear acceleration and angular acceleration/velocity must be available to compensate also dynamic effects. Such accelerations and velocities are estimated by means of a quaternion-based Kalman filter, which is described in the next section, whose only input is a measure of the 6-DOF pose of the robot endeffector (whose orientation is properly transformed into a unit quaternion). First and second order derivatives of the $F / T$ sensor pose $\mathbf{P}_{s}$, generated by the Kalman filter, are used by a model-based load compensation module, processing sensor measurements to extract $\mathbf{F}_{c}$. The overall control scheme is therefore described by the block diagram of Fig. 2. Finally, as is common when admittance control is used, we assume that a low-level position control loop provides accurate tracking of the reference pose, so that $\mathbf{P}_{r e f} \approx \mathbf{P}_{s}$.

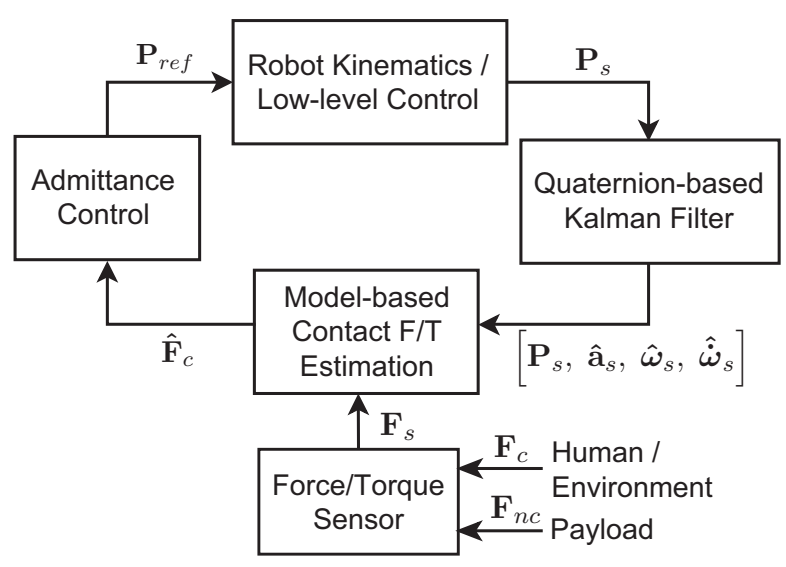

Fig. 2. Block diagram of the proposed interaction control scheme 
Remark 1 The proposed control scheme allows the so-called walk-through programming or hand guidance, implemented on industrial robots by other authors [15] and by companies like FANUC (i.e. by adding the Hand Guidance option to its collaborative robot CR-35iA) or ABB (i.e. by means of its FC Programming Handle add-on). However, these examples require the user to interact with the robot by means of specific handles embedding a F/T sensor, but the latter is not sensitive to the robot payload. In our case, instead, the setup could also be used, when human teaching is concluded, to execute tasks requiring a controlled interaction between payload and environment (e.g. polishing, assembling, etc.).

Remark 2 This paper focuses on the estimation of load accelerations/velocities and the compensation of related effects on F/T measurements, without addressing human safety issues as prescribed by relevant industrial standards (e.g. ISO 10218 [16] and the newer ISO/TS 15066 [17]). While some safety-related aspects can already be addressed in the proposed setup, namely velocity limitation by using the method described in [15], the absence of dangerous collisions between the human and any other link of the robot cannot be guaranteed by only measuring contact $F / T$ on the robot tip. However, even though achieving full compliance to such regulations for standard (i.e. large and non-backdrivable) industrial robots is an issue, we also believe that it could be solved in a near future by using $3 D$ or RGB-D cameras [18], [19], or tactile sensing skins [20].

\section{QUATERNION-BASED KALMAN FILTER}

\section{A. Quaternions}

Several methods can be used to represent the orientation of a rigid body relative to a fixed frame [21]. Among these, quaternions offer a noteworthy solution avoiding the singularity problem of Euler angles and reducing, at the same time, the redundant number of parameters included in a full rotation matrix $\mathbf{R}$. A unit quaternion $\mathbf{q}$ is a four-dimensional vector (with unitary 2-norm) consisting of two parts: a scalar $q_{w}$ and an inner vector $\mathbf{q}_{v}=\left[q_{x}, q_{y}, q_{z}\right]^{T}$. Unit quaternions are strictly related to the axis-angle representation, in which two subcomponents denote respectively the angle $\theta$ and the axis versor $\mathbf{u}$ around which the orientation of the rigid body is defined:

$$
\mathbf{q}=\left[\begin{array}{l}
q_{w} \\
\mathbf{q}_{v}
\end{array}\right]=\left[\begin{array}{c}
\cos \left(\frac{\theta}{2}\right) \\
\mathbf{u} \sin \left(\frac{\theta}{2}\right)
\end{array}\right]
$$

A set of useful operations applied to quaternions can be defined, and they are briefly recalled in the following. More details about quaternion properties can be found in [9].

The algebraic sums of quaternions, as well as the multiplications of a scalar for a quaternion, do not differ from the standard vector operations, whilst the product of two quaternions $\mathbf{q}, \mathbf{r}$ is defined by the non-commutative operator $\otimes$ in terms of cross and dot products among their components:

$$
\mathbf{q} \otimes \mathbf{r}=\left[\begin{array}{c}
q_{w} r_{w}-\mathbf{q}_{v} \cdot \mathbf{r}_{v} \\
\mathbf{q}_{v} \times \mathbf{r}_{v}+q_{w} \mathbf{r}_{v}+r_{w} \mathbf{q}_{v}
\end{array}\right]
$$

Furthermore, considering that the quaternion can be seen as a hyper-complex number, the exponential of a quaternion is an extension of the Euler expression for the exponential of an imaginary number $e^{j \theta}=\cos (\theta)+j \sin (\theta)$ :

$$
e^{\mathbf{q}}=\left\{\begin{array}{cc}
e^{q_{w}}\left[\begin{array}{c}
\cos \left(\left\|\mathbf{q}_{v}\right\|\right) \\
\frac{\mathbf{q}_{v}}{\left\|\mathbf{q}_{v}\right\|} \sin \left(\left\|\mathbf{q}_{v}\right\|\right)
\end{array}\right] & \text { if }\left\|\mathbf{q}_{v}\right\| \neq 0 \\
{[1, \mathbf{0}]^{T}} & \text { otherwise }
\end{array}\right.
$$

where $\|\cdot\|$ is the 2-norm operator. To avoid numerical issues, practical software implementations of the previous formula should consider as zero values of $\left\|\mathbf{q}_{v}\right\|<\varepsilon$, for a properly chosen $\varepsilon$. The exponential of a quaternion can also be expressed as an absolutely convergent series. In case of quaternions characterized by a scalar part equal to zero (pure quaternions), this formulation reduces to:

$$
e^{\mathbf{q}}=\sum_{k=0}^{\infty} \frac{\mathbf{q}^{k}}{k !}
$$

with $\mathbf{q}=\left[0, q_{x}, q_{y}, q_{z}\right]^{T}$ and the power operator understood as successive quaternion products. Another remarkable property of the exponential expression is its relationship with the axisangle meaning of a quaternion, defined in Eq. 2. Indeed, any quaternion $\mathbf{q}$, featuring a rotation around the versor $\mathbf{u}$ by an angle $\theta$, corresponds to the exponential form of:

$$
\mathbf{q}=e^{\frac{\theta \mathbf{u}}{2}}
$$

\section{B. Quaternion Kinematics}

The time variation of the orientation is associated to the time derivative of the unit quaternion describing the orientation of the rigid body. Furthermore, the evolution of that quaternion in time is related to the body angular rate, through the equations:

$$
\dot{\mathbf{q}}=\frac{1}{2} \mathbf{q} \otimes \boldsymbol{\omega}_{b f}=\frac{1}{2} \boldsymbol{\omega}_{w f} \otimes \mathbf{q}
$$

where the body angular rates $\boldsymbol{\omega}_{b f}$ and $\boldsymbol{\omega}_{w f}$ are seen respectively from the body-fixed frame and from the world-fixed frame, and they are considered as pure quaternions $\boldsymbol{\omega}=$ $\left[0, \omega_{x}, \omega_{y}, \omega_{z}\right]^{T}$. It is worth noting that the pure quaternion associated to the angular rate may not have unitary 2-norm.

Consequently, the second-order derivative of the quaternion can be formulated as:

$$
\ddot{\mathbf{q}}=\frac{1}{2}\left(\dot{\mathbf{q}} \otimes \boldsymbol{\omega}_{b f}+\mathbf{q} \otimes \dot{\boldsymbol{\omega}}_{b f}\right)=\frac{1}{2}\left(\dot{\boldsymbol{\omega}}_{w f} \otimes \mathbf{q}+\boldsymbol{\omega}_{w f} \otimes \dot{\mathbf{q}}\right)
$$

In the following, in order to derive a model for the Kalman filter, the time is discretized and the angular acceleration is supposed to be constant between two consecutive time steps. Both the angular variables are seen from the body-fixed frame. Then, the discrete-time integration of the quaternion has to be defined. The starting point is the Taylor series of $\mathbf{q}_{k+1}$ that can be written as:

$$
\mathbf{q}_{k+1}=\mathbf{q}_{k}+\dot{\mathbf{q}}_{k} \Delta t+\frac{1}{2} \ddot{\mathbf{q}}_{k} \Delta t^{2}+\frac{1}{6} \dddot{\mathbf{q}}_{k} \Delta t^{3}+\ldots
$$

where $k$ is the current time step and $\Delta t$ is the sample time.

A first approach to the computation of $\mathbf{q}_{k+1}$ consists in neglecting the terms of order higher than the second, as the successive powers of the sample time commonly become close to zero, and in substituting in Eq. 9 the first and second order derivative of Eq. 7 and Eq. 8. However, such 
integration approach does not preserve the unitary 2-norm of the quaternion, so that a consequent division by $\left\|\mathbf{q}_{k+1}\right\|$ is required in case the integration law is used as a predictor of orientation.

A proper solution overcoming this problem exploits the exponential of quaternions, as reported in [22]. Indeed, because of the previous assumptions, the pure quaternion of the average angular rate $\bar{\omega}$ between two consecutive time steps is given by:

$$
\overline{\boldsymbol{\omega}}_{k}=\boldsymbol{\omega}_{k}+\frac{1}{2} \dot{\boldsymbol{\omega}}_{k} \Delta t
$$

that can be used to expand the angular rate powers:

$$
\begin{gathered}
\boldsymbol{\omega}_{k}=\overline{\boldsymbol{\omega}}_{k}-\frac{1}{2} \dot{\boldsymbol{\omega}}_{k} \Delta t \\
\boldsymbol{\omega}_{k}^{2}=\overline{\boldsymbol{\omega}}_{k}^{2}-\frac{1}{2} \Delta t \overline{\boldsymbol{\omega}}_{k} \otimes \dot{\boldsymbol{\omega}}_{k}-\frac{1}{2} \Delta t \dot{\boldsymbol{\omega}}_{k} \otimes \overline{\boldsymbol{\omega}}_{k}+\frac{1}{4} \Delta t^{2} \dot{\boldsymbol{\omega}}_{k}^{2} \\
\boldsymbol{\omega}_{k}^{3}=\overline{\boldsymbol{\omega}}_{k}^{3}+\ldots
\end{gathered}
$$

Then, substituting the $\boldsymbol{\omega}_{k}$ powers into the quaternion derivatives, and the quaternion derivatives into the discretetime quaternion integration of Eq. 9, the following expression is derived:

$$
\begin{aligned}
\mathbf{q}_{k+1}=\mathbf{q}_{k} \otimes\left(1+\frac{1}{2} \Delta t \overline{\boldsymbol{\omega}}_{k}\right. & \left.+\frac{1}{2}\left(\frac{1}{2} \Delta t \overline{\boldsymbol{\omega}}_{k}\right)^{2}+\ldots\right)+ \\
& +\mathbf{q}_{k} \otimes(\ldots) \Delta t^{3}+\ldots
\end{aligned}
$$

Finally, the first term corresponds to the Taylor series of the exponential of the pure quaternion $\frac{1}{2} \overline{\boldsymbol{\omega}}_{k} \Delta t$ (see Eq. 5) and the successive terms can be neglected, obtaining:

$$
\mathbf{q}_{k+1}=\mathbf{q}_{k} \otimes e^{\frac{1}{2}\left(\boldsymbol{\omega}_{k} \Delta t+\frac{1}{2} \dot{\boldsymbol{\omega}}_{k} \Delta t^{2}\right)}
$$

where the exponential factor represents the angular increment in the orientation of a quantity equal to $\boldsymbol{\omega}_{k} \Delta t+\frac{1}{2} \dot{\boldsymbol{\omega}}_{k} \Delta t^{2}$, as pointed out previously (see Eq. 6). This integration formula preserves the unitary 2-norm of the quaternion. An alternative formula, more complex but possibly more accurate for a large $\Delta t$, can be found in [23].

\section{Kalman Filter Model}

Based on the assumption that the only available measurement is the output of robot forward kinematics, two Kalman filters have been designed in order to estimate both the linear and the angular variables (velocity and acceleration) of the load: the former is a classic Kalman filter that accomplishes the main task of the estimation of the linear acceleration, while the latter is an extended Kalman filter (EKF) aimed at producing an estimation of the load angular variables.

The general scheme of a Kalman filter used for differentiation assumes the following dynamic model for the state $\mathbf{x}$ and the measurement $\mathbf{y}$ :

$$
\begin{aligned}
& \mathbf{x}_{k+1}=\mathbf{A} \mathbf{x}_{k}+\mathbf{w}_{k} \\
& \mathbf{y}_{k}=\mathbf{C} \mathbf{x}_{k}+\boldsymbol{\nu}_{k}
\end{aligned}
$$

where $\mathbf{A}$ and $\mathbf{C}$ are constant matrices, $\mathbf{w}$ is the process noise with covariance matrix $\mathbf{Q}$ and $\boldsymbol{\nu}$ is the measurement noise with covariance matrix $\mathbf{R}$. In differentiation applications, it is generally assumed that the process noise on the higher order derivative is the largest, while the measurement is assumed to be reliable and, therefore, its covariance is low.
The filtering loop consists of two phases: the former accomplishes the prediction of the next state vector $\hat{\mathbf{x}}_{k+1}^{-}$by means of Eq. 14 (with no noise applied) and the prediction of the error covariance matrix $\mathbf{P}_{k+1}^{-}$:

$$
\begin{gathered}
\hat{\mathbf{x}}_{k+1}^{-}=\mathbf{A} \hat{\mathbf{x}}_{k} \\
\mathbf{P}_{k+1}^{-}=\mathbf{A} \mathbf{P}_{k} \mathbf{A}^{T}+\mathbf{Q}
\end{gathered}
$$

Then, the second phase performs a correction of the predicted variables based on the Kalman gain $\mathbf{K}_{k}$, computed as follows:

$$
\begin{gathered}
\mathbf{K}_{k}=\mathbf{P}_{k+1}^{-} \mathbf{C}^{T}\left(\mathbf{C} \mathbf{P}_{k+1}^{-} \mathbf{C}^{T}+\mathbf{R}\right)^{-1} \\
\hat{\mathbf{x}}_{k+1}=\hat{\mathbf{x}}_{k+1}^{-}+\mathbf{K}_{k}\left(\mathbf{y}_{k+1}-\mathbf{C} \hat{\mathbf{x}}_{k+1}^{-}\right) \\
\mathbf{P}_{k+1}=\left(\mathbf{I}-\mathbf{K}_{k} \mathbf{C}\right) \mathbf{P}_{k+1}^{-}
\end{gathered}
$$

This general structure is applied to the linear filter proposed in this work, in which the state vector $\mathbf{x}_{l}$ contains the position $\mathbf{p}$, the linear velocity $\mathbf{v}$ and the linear acceleration $\mathbf{a}$ :

$$
\mathbf{x}_{l}=\left[\begin{array}{l}
\mathbf{p} \\
\mathbf{v} \\
\mathbf{a}
\end{array}\right]=\left[x, y, z, v_{x}, v_{y}, v_{z}, a_{x}, a_{y}, a_{z}\right]^{T}
$$

In this case, the evolution law exploits the Euler integration, assuming constant acceleration during two consecutive time steps. Therefore, the prediction step is accomplished by means of the following dynamic matrix:

$$
\mathbf{A}_{l}=\left[\begin{array}{ccc}
\mathbf{I}_{3 \times 3} & \Delta t \mathbf{I}_{3 \times 3} & \frac{1}{2} \Delta t^{2} \mathbf{I}_{3 \times 3} \\
\mathbf{0}_{3 \times 3} & \mathbf{I}_{3 \times 3} & \Delta t \mathbf{I}_{3 \times 3} \\
\mathbf{0}_{3 \times 3} & \mathbf{0}_{3 \times 3} & \mathbf{I}_{3 \times 3}
\end{array}\right]
$$

where $\mathbf{I}$ is the identity matrix and $\mathbf{0}$ is the matrix of zeros. The available measurements $\mathbf{y}_{l}$ for the correction step concern the load position, extracted from robot kinematics, so that:

$$
\mathbf{y}_{l}=\mathbf{C}_{l} \mathbf{x}_{l}=\left[\begin{array}{ll}
\mathbf{I}_{3 \times 3} & \mathbf{0}_{3 \times 6}
\end{array}\right] \mathbf{x}_{l}
$$

The EKF scheme exploited for the estimation of the angular variables differs from the general structure in terms of the state evolution part of Eq. 14, that is replaced by a nonlinear function $\mathbf{f}$ :

$$
\mathbf{x}_{k+1}=\mathbf{f}\left(\mathbf{x}_{k}\right)+\mathbf{w}_{k}
$$

Regarding the other filter equations, the matrix $\mathbf{A}$ is replaced by the Jacobian matrix $\mathbf{F}$, with:

$$
\mathbf{F}=\left.\frac{\partial \mathbf{f}}{\partial \mathbf{x}}\right|_{\mathbf{x}=\hat{\mathbf{x}}}
$$

In more details, the state vector $\mathbf{x}_{a}$ of the angular filter contains the quaternion representing the orientation of the load, as well as the angular velocity and the angular acceleration in the form of pure quaternions (scalar parts are omitted):

$$
\mathbf{x}_{a}=\left[\begin{array}{c}
\mathbf{q} \\
\boldsymbol{\omega} \\
\dot{\boldsymbol{\omega}}
\end{array}\right]=\left[q_{w}, q_{x}, q_{y}, q_{z}, \omega_{x}, \omega_{y}, \omega_{z}, \dot{\omega}_{x}, \dot{\omega}_{y}, \dot{\omega}_{z}\right]^{T}
$$

The prediction step of the filter replaces Eq. 15 with a nonlinear one based on $\mathbf{f}$, that in this case includes the exponential integration of Eq. 13, while the angular velocity and 
the angular acceleration follow the Euler integration formula, that is linear in the state variables:

$$
\left[\begin{array}{c}
\hat{\mathbf{q}}_{k+1}^{-} \\
\hat{\boldsymbol{\omega}}_{k+1}^{-} \\
\hat{\dot{\boldsymbol{\omega}}}_{k+1}^{-}
\end{array}\right]=\left[\begin{array}{c}
\hat{\mathbf{q}}_{k} \otimes e^{\frac{1}{2}\left(\hat{\boldsymbol{\omega}}_{k} \Delta t+\frac{1}{2} \hat{\boldsymbol{\omega}}_{k} \Delta t^{2}\right)} \\
\hat{\boldsymbol{\omega}}_{k}+\hat{\dot{\boldsymbol{\omega}}}_{k} \Delta t \\
\dot{\hat{\boldsymbol{\omega}}}_{k}
\end{array}\right]
$$

The correction step of the filter involves the acquisition of the orientation measurements from the system, expressed as unit quaternions. They are grouped into the vector $\mathbf{y}_{a}$ and related to the state by means of the matrix $\mathbf{C}_{a}$ :

$$
\mathbf{y}_{a}=\mathbf{C}_{a} \mathbf{x}_{a}=\left[\begin{array}{ll}
\mathbf{I}_{4 \times 4} & \mathbf{0}_{4 \times 6}
\end{array}\right] \mathbf{x}_{a}
$$

More details about the initialization of the covariance matrices $\mathbf{Q}_{l}, \mathbf{R}_{l}, \mathbf{Q}_{a}$, and $\mathbf{R}_{a}$, will be given in Sec. V.

\section{LOAD COMPENSATION AND HUMAN-INPUT DETECTION}

Model-based compensation of non-contact F/T contributions from the sensor measurements requires, in addition to the dynamic estimates provided by the Kalman filter described in previous section, an accurate knowledge of mass, COM and inertia tensor of the load. In this paper, whose focus is on Kalman filter design and human-robot interaction, we assume that such parameters are known in advance. Practical applications in a real industrial environment would require online calibration of the load features, as described in [24] and more recently in [25], using methods that can be straighforwardly integrated in the framework proposed here. The rest of the section describes the computational process for contact F/T estimation and introduce additional steps required for human input detection.

The raw measurement $\mathbf{F}_{s}$ from an $\mathrm{F} / \mathrm{T}$ sensor mounted as shown in Fig. 1 includes a sum of three terms: the noncontact one $\mathbf{F}_{n c}=\left[\mathbf{f}_{n c}, \boldsymbol{\tau}_{n c}\right]^{T}$, the contact one $\mathbf{F}_{c}$ and an offset $\mathbf{F}_{o}$. The latter is a temperature-dependent contribution, typical of strain gage-based devices, that can be usually zeroed periodically using a tare function embedded in the F/T sensor. Knowing the mass of the load $m$, the location of its COM $\mathbf{c}_{s}=\left[c_{x}^{s}, c_{y}^{s}, c_{z}^{s}\right]$ (w.r.t. the $\mathrm{F} / \mathrm{T}$ sensor frame), its $3 \times 3$ inertia tensor $\mathbf{I}_{s}$ (as seen from the $\mathrm{F} / \mathrm{T}$ sensor frame) and, of course, velocities/accelerations of the F/T sensor frame, the non-contact term can be computed as follows [7]:

$$
\left[\begin{array}{c}
\mathbf{f}_{n c} \\
\boldsymbol{\tau}_{n c}
\end{array}\right]=\left[\begin{array}{c}
m\left(\mathbf{a}_{s}-\mathbf{g}_{s}\right)+\dot{\boldsymbol{\omega}}_{s} \times m \mathbf{c}_{s}+\boldsymbol{\omega}_{s} \times\left(\boldsymbol{\omega}_{s} \times m \mathbf{c}_{s}\right) \\
\mathbf{I}_{s} \dot{\boldsymbol{\omega}}_{s}+\boldsymbol{\omega}_{s} \times \mathbf{I}_{s} \boldsymbol{\omega}_{s}+m \mathbf{c}_{s} \times \mathbf{a}_{s}-m \mathbf{c}_{s} \times \mathbf{g}_{s}
\end{array}\right]
$$

The previous equation can be reworked to highlight its linear dependency from the load inertial parameter vector $\mathbf{\Phi}_{l}^{s}=\left[m, m c_{x}^{s}, m c_{y}^{s}, m c_{z}^{s}, I_{x x}^{s}, I_{x y}^{s}, I_{x z}^{s}, I_{y y}^{s}, I_{y z}^{s}, I_{z z}^{s}\right]^{T}$, obtaining the matrix form:

$$
\mathbf{F}_{n c}=\mathbf{V}_{s}\left(\mathbf{a}_{s}, \boldsymbol{\omega}_{s}, \dot{\boldsymbol{\omega}}_{s}, \mathbf{g}_{s}\right) \Phi_{l}^{s}
$$

in which $\mathbf{V}_{s}(\ldots)$ is a $6 \times 10$ matrix including velocities/accelerations and elements of the gravity vector $\mathbf{g}_{s}$, expressed in the current $\mathrm{F} / \mathrm{T}$ sensor frame.

If the load cannot be detached easily from the end-effector, force and torques of the offset term $\left[\mathbf{f}_{o}, \boldsymbol{\tau}_{o}\right]^{T}$ can still be eliminated with the help of Eq. 29. In fact, if the loaded F/T sensor is zeroed in a static initial condition with a known orientation, then it is necessary to add to subsequent readings a pseudo-gravitational term $\mathbf{F}_{g_{\text {init }}}=\mathbf{V}_{s}\left(\mathbf{0}, \mathbf{0}, \mathbf{0}, \mathbf{g}_{s_{\text {init }}}\right) \boldsymbol{\Phi}_{l}^{s}$. This offset zeroing operation should also be repeated periodically, during a pause of the robotic task, to cope with temperature variations and slow drifts.

The computation of contact F/T is based on uncertain quantities, namely load parameters, whether they are determined offline or estimated online, and estimates from the Kalman filter. Therefore, its expression should be written as follows:

$$
\hat{\mathbf{F}}_{c}=\mathbf{F}_{s}-\hat{\mathbf{F}}_{n c}+\hat{\mathbf{F}}_{g_{i n i t}}
$$

Because of such uncertainties, it is necessary to define a threshold-based logic for the actual contact detection. Assuming that all of the 19 uncertain variables are grouped in the vector $\Theta=\left[\hat{\mathbf{a}}_{s}, \hat{\boldsymbol{\omega}}_{s}, \hat{\dot{\boldsymbol{\omega}}}_{s}, \hat{\mathbf{\Phi}}_{l}^{s}\right]^{T}$ and that each element $\Theta_{j}$ of the vector is affected by an uncertainty $\Delta \Theta_{j}$, the propagation of uncertainties in the nonlinear function of Eq. 29 can be written as follows, denoting with $F_{\bullet}^{i}$ the $i$-th component of a 6D F/T vector (i.e. $F_{\bullet}^{1}=f_{x_{\bullet}}, F_{\bullet}^{4}=\tau_{x_{\bullet}}$ ):

$$
\Delta \hat{F}_{n c}^{i}=\sqrt{\sum_{j=1}^{19}\left(\frac{\partial F_{n c}^{i}}{\partial \Theta_{j}}\right)^{2}\left(\Delta \Theta_{j}\right)^{2}}
$$

In the case of the variables estimated with Kalman filtering, the uncertainty can be defined (neglecting correlations) as the square root of the diagonal terms in the error covariance matrix $\mathbf{P}$, multiplied by 2 to obtain a $95 \%$ confidence level. In the case of load parameters, unless they are obtained with an online identification procedure (that would provide a similar information about their variance), an arbitrary confidence level can be specified. Finally, the adaptive threshold can be defined, with an additional and constant safety margin $\mathbf{F}_{\text {min }}$, as follows:

$$
\mathbf{F}_{t h}=\Delta \hat{\mathbf{F}}_{n c}+\mathbf{F}_{\text {min }}
$$

Another important aspect that must be taken into account for the purpose of human-input detection is the spectral content of the contact signal. Several authors pointed out that intentional human stimuli have the most dominant frequency components within a band from 0 to at most $5 \mathrm{~Hz}$ (possibly less), while accidental collisions can be commonly detected as highfrequency (i.e. higher than $10 \mathrm{~Hz}$ ) peaks [5], [4], [3]. Since our aim is to directly exploit the former components as an input to the admittance control scheme of Fig. 2, we assume that the output of Eq. 30 is further processed by a low-pass filter to obtain $\hat{\mathbf{F}}_{c_{f}}$, as an evaluation of the human intentional contact. Different tunings (i.e. with higher cutoff frequencies), of this low-pass filter could eventually be necessary for the execution of other tasks, involving autonomous behavior and robot-environment contacts. In the latter case, especially when stiff enviroments are considered, tuning of the filter would also influence the stability of the contact transition behavior. However, this issue has not been addressed so far in our experiments. 
Finally, the threshold-based evaluation of the filtered contact estimation for human-input detection can be written, for each component of the F/T vector, as follows:

$$
\hat{F}_{c_{h}}^{i}= \begin{cases}\hat{F}_{c_{f}}^{i}-F_{t h}^{i} & \text { if } \hat{F}_{c_{f}}^{i}>F_{t h}^{i} \\ \hat{F}_{c_{f}}^{i}+F_{t h}^{i} & \text { if } \hat{F}_{c_{f}}^{i}<-F_{t h}^{i} \\ 0 & \text { otherwise }\end{cases}
$$

\section{EXPERIMENTAL RESULTS}

The proposed control scheme has been implemented using the Orocos [26] real-time framework and tested on a KUKA LWR 4+ (workspace radius $800 \mathrm{~mm}$, rated payload $7 \mathrm{~kg}$ ), equipped with a 6-DOF F/T ATI Mini45 IP65 (measuring range $\pm 145 \mathrm{~N}$ on $\mathrm{X}-\mathrm{Y}, \pm 290 \mathrm{~N}$ on $\mathrm{Z}, \pm 5 \mathrm{Nm}$ on all axes, resolution $1 / 16 \mathrm{~N}-1 / 752 \mathrm{Nm}$ ). It is important to remark that even if the KUKA controller directly supports compliant control modes (i.e. Cartesian or joint impedance), in this context only direct/inverse kinematics and joint space (noncompliant) position control functionalities have been used, in order to emulate the features of a common industrial robot with stiff low-level control.

\section{A. Kalman filter evaluation}

The first tests were made to evaluate the computational efficiency and the reliable convergence of the quaternionbased Kalman filter. The latter is crucial to preserve a stable interaction behavior of the proposed admittance controlled robotic system. The full implementation of the linear and angular estimation, using the Eigen library [27], requires a mean execution time of $408 \mu \mathrm{s}$ on a PC with an Intel $\mathrm{R}$ Core $^{T M}$ i5$2300 \mathrm{CPU} @ 2.80 \mathrm{GHz} \times 4,8 \mathrm{~Gb}$ RAM and Ubuntu $14.04 \mathrm{OS}$. Moreover, the EKF did not show any convergence issue with sampling times of up to $100 \mathrm{~ms}$, even though the quality of the estimate is acceptable for our load compensation purposes only with sampling times up to $10 \mathrm{~ms}$. During the reported experiments, the Kalman filter rate was fixed to $500 \mathrm{~Hz}$ (the same sampling frequency was used for F/T measurements and admittance control). Moreover, after proper tuning to achieve a good tradeoff between dynamic response and output smoothness, the covariance matrices of process noise were set to $\mathbf{Q}_{l}=\operatorname{diag}\left(0,0,0,10^{-6}, 10^{-6}, 10^{-6}, 10^{-2}, 10^{-2}, 10^{-2}\right)$ and $\mathbf{Q}_{a}=\operatorname{diag}\left(0,0,0,0,10^{-6}, 10^{-6}, 10^{-6}, 10^{-2}, 10^{-2}, 10^{-2}\right)$, while those of measurement noise to $\mathbf{R}_{l}=10^{-10} \mathbf{I}_{3 \times 3}$ and $\mathbf{R}_{a}=$ $10^{-10} \mathbf{I}_{4 \times 4}$. This choice follows the rule of thumb described in Sec. III-C.

As shown in Fig. 3 and Fig. 4 the Kalman filter is a mandatory alternative to numerical differentiation (at the same sampling rate), here calculated using the first order derivative operator for homogeneous transformation matrices, called diff () in the Orocos Kinematics Dynamics Library (KDL) [28], and subsequent differentiation of the diff () result to obtain angular accelerations. The latter, in particular, are too noisy to be useful and processing them with an additional filter (in these experiments, a low-pass FIR filter with $20 \mathrm{~Hz}$ cutoff frequency, designed using the Parks-McClellan algorithm, that guarantees equiripple magnitude and linear phase response) is not a viable alternative, because it introduces an undesirable (i.e. in terms of real-time compensation of load dynamics) phase lag, negligible in a properly tuned Kalman filter.

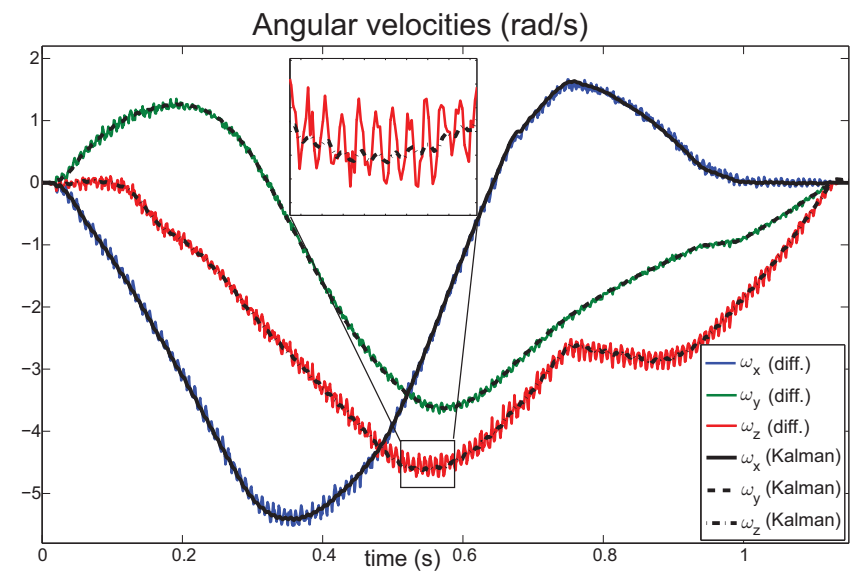

Fig. 3. Comparison between Kalman filter and $\operatorname{diff}()$ : angular velocities (all axes)

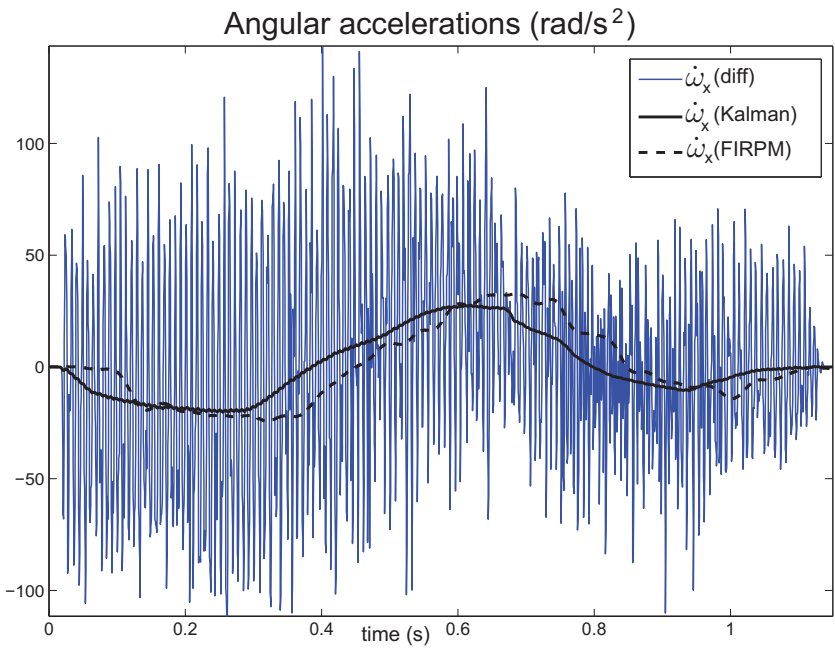

Fig. 4. Comparison among Kalman filter, pure differentiation and differentiation plus a Parks-McClellan FIR filter: angular acceleration ( $\mathrm{X}$ axis)

Several authors describe the use of inertial sensors (i.e. accelerometers for linear accelerations and gyroscopes for angular velocities) to extract information about higher order dynamic variables of a robot [24], [8]. Therefore, we compared the outputs of the proposed Kalman filter with those measured from an Inertial Measurement Unit (IMU), namely a 9DOF Razor by SparkFun Electronics (measuring range $\pm 16 \mathrm{~g}$, 13-bit resolution, on accelerations and $\pm 2000 \mathrm{deg} / \mathrm{s}, 16$-bit resolution, on angular rates), mounted on the end-effector of the KUKA robot. Since the range and resolution of these accelerometers do not allow a fair comparison, only angular rates will be considered in the following. The used IMU embeds digital low-pass filters with configurable cutoff frequency, set to $200 \mathrm{~Hz}$ for gyroscopes. As shown by Fig. 5 the latter sensors provide accurate values, though the Kalman filter output is still smoother, but then angular accelerations should be estimated using again numerical differentiation and amplifying noise. 


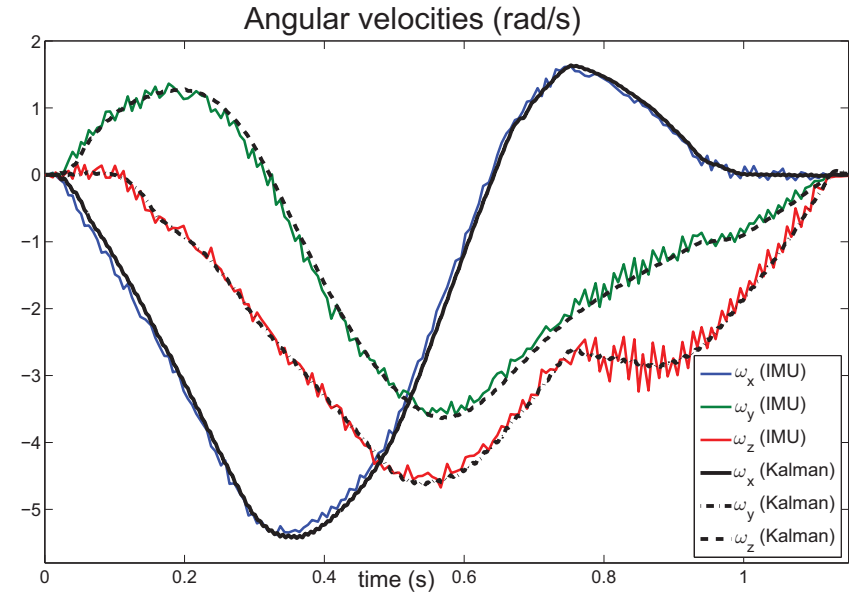

Fig. 5. Comparison between Kalman filter and IMU: angular velocities

\section{B. Effects of load dynamics in absence of contacts}

With regard to the estimation of contact forces, we first tested the computational process of Sec. IV without lowpass filters and thresholds, mounting as load an aluminium cylinder (1.042 kg mass, $3.6 \mathrm{~cm}$ height, $11.5 \mathrm{~cm}$ diameter) and forcing point-to-point free motions (i.e. without contacts with environment or humans) with direct joint space commands. Fig. 6 shows measured forces and estimated contact forces during a motion in which the linear velocity of the load reached $1 \mathrm{~m} / \mathrm{s}$ (four times faster than the limit prescribed by ISO 10216 during a cooperation with a human), with a maximum linear acceleration of $3 \mathrm{~m} / \mathrm{s}^{2}$. As can be seen, estimated contacts are close to zero, so that the non-contact effect is correctly eliminated even during such a fast motion and its acceleration/deceleration phases. In these transients, residual oscillations are visible in the contact estimation, but were not observed in the outputs of the Kalman filter. An explanation of this effect could be related to the structural flexibilities in the robot and especially in the mounting adapters for F/T sensor and load. Since such effects would instead be captured by an IMU mounted near the load, the proposed Kalman filter could be extended to embed fusion of measurements from these sensors, if higher accuracy is required.

During the same free motion tests, the adaptive threshold parameters were tuned and verified. As shown in Fig. 7, the choice of the model-based adaptation mechanism defined by Eq. 32 allows to avoid false positive contact detection, that would instead be possible with a fixed threshold. After such experiments, the modeling uncertainty was fixed to $2 \%$ of the nominal value on all load parameters and the safety margin to $\mathbf{F}_{\text {min }}=[0.4,0.4,0.4,0.1,0.1,0.1]^{T}$.

\section{Human input detection tests}

Finally, the full control scheme has been tested during a manipulation task emulating a human/robot collaboration for transportation of a large object. The task requires the human to push the object carried by the robot and force the latter to compliantly follow the F/T stimuli, as detected by the previously described threshold-based logic. In addition

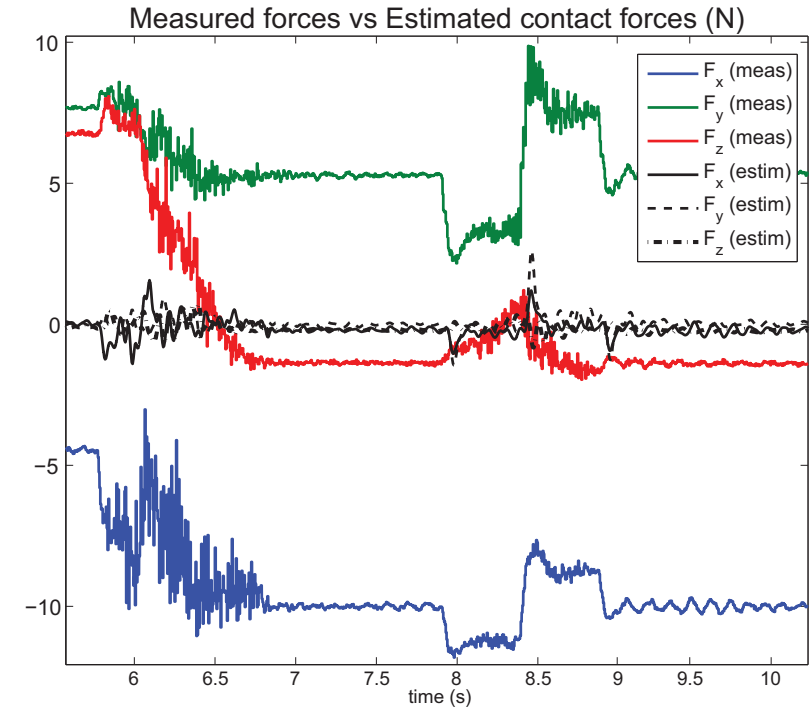

Fig. 6. Comparison between measured forces and estimated contact forces during a free motion (no contacts)

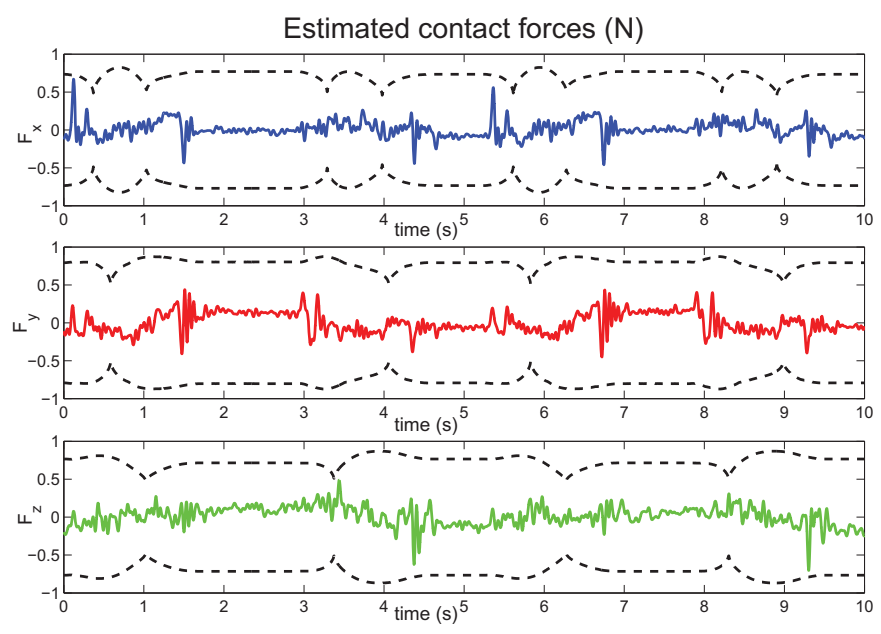

Fig. 7. Estimated contact forces with adaptive thresholds (no contacts)

to the adaptive threshold parameters mentioned above, the contact force estimation has been processed by a 4-th order Butterworth low-pass filter with a $2.5 \mathrm{~Hz}$ cutoff frequency, a band compatible with suggestions taken from the literature [5]. The plot of Fig. 8 shows that the contact force estimate, after filtering and thresholding, provides a smooth input to the admittance controller governed by Eq. 1 during intentional human/robot interactions. Instead, when the load is hit accidentaly by a rigid object, the high-frequency peaks that are visible in the raw contact force estimates are eliminated by the low-pass filter and do not cause abrupt robot motions.

Since the human intent, detected by contact force estimation, is the only input of the mass-damper interaction model, its smoothness guarantees a corresponding smooth behavior of the robot, as can be observed in the accompanying video.

\section{CONCLUSION}

The paper has presented a control framework that is designed to extend pHRI applications on a broader industrial 


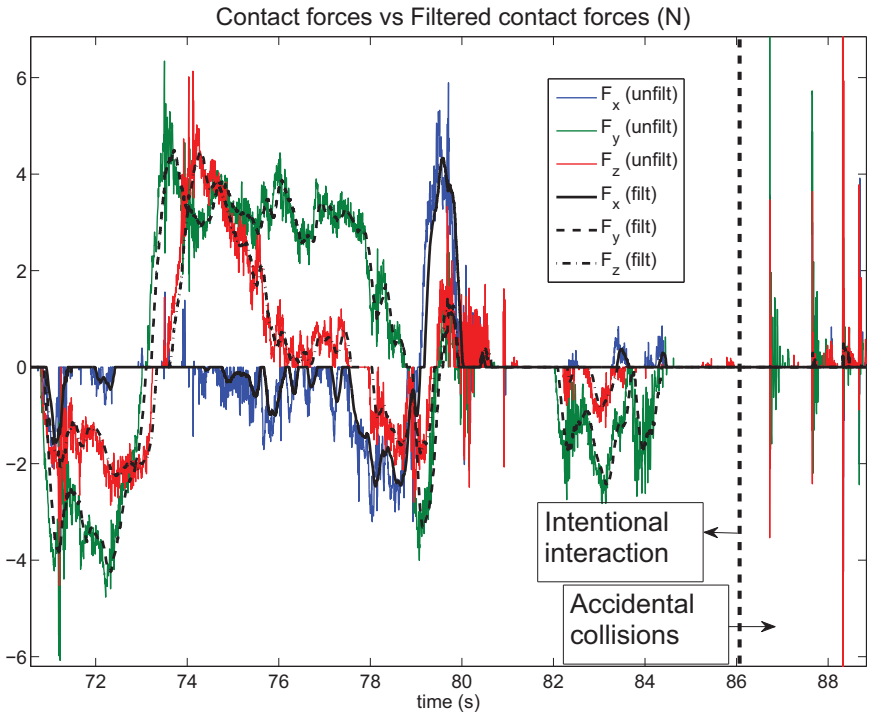

Fig. 8. Contact forces, not filtered (colored lines) and filtered (black lines), during human/robot interaction

context, including plants in which industrial robots with closed and stiff control systems are already installed. The proposed framework is based on admittance control and force/torque $(\mathrm{F} / \mathrm{T})$ sensing of the contact between the robot and its environment, with the specific intent to detect the input from a human user. To compensate the effect of a large payload possibly installed on the end-effector of the robot, the measurements of robot pose, which are always available from any industrial robot controller, are processed by a quaternion-based Kalman filter to estimate the velocities/accelerations of the load.

In future works we will compare the performance of the proposed Kalman filter with those obtained using an Unscented Kalman Filter (UKF), for quaternion processing. Moreover, we aim to characterize more precisely the human intent as detected by the contact force estimation process described in the paper, with the aim to predict the task that he/she wants to infer to the robot and select and adapt predefined motion primitives for their online execution. This feature would better support human operators during the execution of manipulation operations of industrial interest, like pick-and-place or parts assembly. Reconfiguration of the system according to the task and the operating mode (i.e. teaching or autonomous execution), particularly concerning the cutoff frequency of contact F/T filtering, will also be an important part of future experiments.

\section{REFERENCES}

[1] L. Villani and J. De Schutter, "Force control." in Springer Handbook of Robotics, B. Siciliano and O. Khatib, Eds. Springer Berlin Heidelberg, 2008 , ch. 7.

[2] F. D'Ippolito, F. Alonge, and E. Cucco, "Contact estimation in robot interaction," International Journal of Advanced Robotic Systems, vol. 11, no. $96,2014$.

[3] C. Cho and J. Song, "Collision detection algorithm robust to model uncertainty," International Journal of Control, Automation and Systems, vol. 11, no. 4, pp. 776-781, August 2013.

[4] M. Geravand, F. Flacco, and A. De Luca, "Human-robot physical interaction and collaboration using an industrial robot with a closed control architecture," in IEEE International Conference on Robotics and Automation (ICRA), Karlsruhe, Germany, May 2013.

[5] M. Erden and T. Tomiyama, "Human-intent detection and physically interactive control of a robot without force sensors," IEEE Transactions on Robotics, vol. 26, no. 2, April 2010.

[6] F. Aghili, "Robust impedance control of manipulators carrying a heavy payload," Journal of Dynamic Systems, Measurements and Control, vol. 132, no. 5, September 2010.

[7] D. Kubus, T. Kröger, and F. Wahl, "Improving force control performance by computational elimination of non-contact forces/torques," in IEEE International Conference on Robotics and Automation (ICRA), Pasadena, USA, May 2008.

[8] B. Munoz-Barron, J. Rivera-Guillen, R. Osornio-Rios, and R. RomeroTroncoso, "Sensor fusion for joint kinematic estimation in serial robots using encoder, accelerometer and gyroscope," Journal of Intelligent and Robotic Systems, vol. 78, no. 3, pp. 529-540, June 2015.

[9] J. Kuipers, Quaternion and rotation sequences: a primer with applications to orbits, aerospace and virtual reality. Princeton University Press, 1999

[10] A. Sabatini, "Quaternion-based extended Kalman filter for determining orientation by inertial and magnetic sensing," IEEE Transactions on Biomedical Engineering, vol. 53, no. 7, July 2006.

[11] A. Ude, "Filtering in a unit quaternion space for model-based object tracking," Robotics and Autonomous Systems, vol. 28, no. 2-3, pp. $163-$ 172, 1999.

[12] X. Yun and E. Bachmann, "Design, implementation, and experimental results of a quaternion-based Kalman filter for human body motion tracking," IEEE Transactions on Robotics, vol. 22, no. 6, December 2006.

[13] G. Bätz, B. Weber, M. Scheint, D. Wollherr, and M. Buss, "Dynamic contact force/torque observer: sensor fusion for improved interaction control," International Journal of Robotics Research, vol. 32, no. 4, pp. 446-457, 2013.

[14] V. Duchaine and C. Gosselin, "Investigation of human-robot interaction stability using Lyapunov theory," in IEEE International Conference on Robotics and Automation (ICRA), Pasadena, USA, May 2008.

[15] L. Bascetta, G. Ferretti, G. Magnani, and P. Rocco, "Walk-through programming for robotic manipulators based on admittance control," Robotica, vol. 31, no. 7, pp. 1143-1153, October 2013.

[16] "ISO 10218-1:2011 Robots for Industrial Environments: Safety Requirements (Part 1: Robot)," International Organization for Standardization, Standard, 2011.

[17] "ISO/TS 15066:201 Robots and robotic devices: Collaborative robots," International Organization for Standardization, Standard, 2016.

[18] P. Rybski, P. Anderson-Sprecher, D. Huber, C. Niessl, and R. Simmons, "Sensor fusion for human safety in industrial workcells," in 2012 IEEE/RSJ International Conference on Intelligent Robots and Systems, Oct 2012, pp. 3612-3619.

[19] E. Magrini, F. Flacco, and A. De Luca, "Estimation of contact forces using a virtual force sensor," in IEEE/RSJ International Conference on Intelligent Robots and Systems (IROS), Chicago, USA, Sept. 2014.

[20] J. O'Neill, J. Lu, R. Dockter, and T. Kowalewski, "Practical, stretchable smart skin sensors for contact-aware robots in safe and collaborative interactions," in IEEE International Conference on Robotics and Automation (ICRA), May 2015.

[21] J. Diebel, "Representing attitude: Euler angles, unit quaternions, and rotation vectors," Matrix, vol. 58, no. 15-16, pp. 1-35, 2006.

[22] J. Solà, "Quaternion kinematics for the error-state KF," IRI, Universitat Politècnica de Catalunya, Barcelona, Spain, Tech. Rep., 2016.

[23] F. Zhao and B. van Wachem, "A novel quaternion integration approach for describing the behaviour of non-spherical particles," Acta Mechanica, vol. 224 , no. 12,2013

[24] D. Kubus, T. Kröger, and F. Wahl, "On-line estimation of inertial parameters using a recursive total least-squares approach," in IEEE/RSJ International Conference on Intelligent Robots and Systems (IROS), Nice, France, Sept. 2008.

[25] S. Traversaro, S. Brosette, A. Escande, and F. Nori, "Identification of fully physical consistent inertial parameters using optimization on manifolds," in IEEE/RSJ International Conference on Intelligent Robots and Systems (IROS), Daejeon, Korea, Oct. 2016.

[26] The Orocos Project, "The Orocos Toolchain and Real-Time Toolkit," http://www.orocos.org/toolchain.

[27] G. Guennebaud, B. Jacob et al., "Eigen v3," http://eigen.tuxfamily.org, 2010.

[28] R. Smits, "KDL: Kinematics and Dynamics Library," http://www.orocos.org/kdl. 